\title{
Cryptanalysis of Two User Identification Schemes with Key Distribution Preserving Anonymity
}

\author{
Eun-Jun Yoon and Kee-Young Yoo* \\ Department of Computer Engineering, Kyungpook National University, \\ Daegu 702-701, South Korea \\ Tel.: +82-53-950-5553; Fax: +82-53-957-4846 \\ ejyoon@infosec.knu.ac.kr, yook@knu.ac.kr
}

\begin{abstract}
In 2004, Wu-Hsu proposed an efficient identification scheme preserving anonymity. However, Yang et al. showed that Wu-Hsu's scheme has a serious weakness, by which the service provider can learn the secret token of the user who requests services. To overcome this limitation, they further proposed a scheme to attain the same set of objectives as the previous works. Nevertheless, the two schemes still have other serious weaknesses. Accordingly, the current paper demonstrates the vulnerability of the two schemes. Furthermore, we present a method to avoid attack.
\end{abstract}

Keywords: Cryptography, Password, Key establishment, Forward Secrecy.

\section{Introduction}

In distributed computing environments, it is necessary to maintain user anonymity. That is, only the service provider can identify the user, while no other entity can determine any information concerning the user's identity. In 2000, Lee and Chang 1 proposed a user identification scheme based on the security of the factoring problem [2] 3] and the one-way hash function [3] 4]. Their scheme has the following advantages: (1) Users can request services without revealing their identities to the public; (2) Each user needs to maintain only one secret; (3) It is not necessary for service providers to record the password files for the users; (4) No master key updating is needed if a new service provider is added into the system.

However, in 2004, Wu-Hsu (WH) [5] showed that Lee-Chang's user identification scheme is insecure under two attacks. First, when a user requests service from a service provider, since only one-way authentication of the user is implemented, an attacker can impersonate the service provider; second, if an expired session key is disclosed, an attacker can break the user anonymity of the corresponding past session. Then they proposed a more efficient identification scheme

\footnotetext{
* Corresponding author.
} 
preserving the same merits [5]. The WH scheme not only effectively eliminates the security leaks of the Lee-Chang scheme, it also reduces computational complexities and communication costs.

Recently, Yang et al. (YWBWD) [6] showed that the WH scheme has a serious weakness, by which the service provider can learn the secret token of the user who requests services. To overcome this limitation, they further proposed a scheme to attain the same set of objectives as the previous works.

However, the WH scheme and YWBWD scheme have other serious weaknesses. Accordingly, the current paper demonstrates the vulnerability of two schemes. Using our attacks, we will show that a malicious user (including the service provider) can easily obtain a specific legal user's secret token and impersonate this specific user to request a service from the service provider and gain access privilege. Additionally, we will show that a malicious user (including the legal user) can easily get the service provider's secret token and impersonate this service provider to exchange a common session key with a legal user. Furthermore, we present an improvement to repair the security flaws of the two schemes.

This paper is organized as follows: In Section 2, we briefly review the WH scheme and YWBWD scheme. Section 3 shows the security flaws of two schemes. In Section 4, we present an improvement of the two schemes. In Section 5, we analyze the security of our proposed scheme. Finally, our conclusions are presented in Section 6.

\section{Literature Review}

This section separately reviews the WH scheme [5] and YWBWD scheme [6].

\subsection{WH Scheme}

The WH scheme is composed of two phases: key generation and anonymous user identification.

Key Generation Phase: The key generation phase of the WH scheme, which is illustrated in Figure 1, is as follows: A smart card producing center $(S C P C)$ first chooses two large primes $p$ and $q$, computes $N=p q$, picks an element $g \in Z_{N}^{*}$ and a hash function $h(\cdot)$, and selects $e$ and $d$ such that $e d=1(\bmod \phi(N))$, where $\phi(N)(=(p-1)(q-1))$ is the Euler totient function. $N, e, g$ and $h(\cdot)$ are published and $d, p$, and $q$ are kept secret by $S C P C$. Then, $S C P C$ sends each user $U_{i}$ (or service provider $P_{i}$ ) a secret token $S_{i}$ with a secure channel, where $S_{i}=I D_{i}^{d}(\bmod N)$ and $I D_{i}$ is the identity of $U_{i}\left(\right.$ or $\left.P_{i}\right)$.

Anonymous User Identification Phase: The anonymous user identification phase of WH scheme, as illustrated in Figure 2, is as follows:

(1) $U_{i}$ first submits a service request to $P_{j}$ to request a service from the service provider $P_{j}$. 


\section{$S C P C$}

Choose: $p, q$

Compute: $N=p q$

Pick: $g \in Z_{N}^{*}, h(\dot{)}$

Compute: $\phi(N)=(p-1)(q-1)$

Select: $e, d(e d=1(\bmod \phi(N)))$

Publish: $N, e, g, h(\cdot)$

Keep secret: $d, p, q$

Compute: $S_{i}=I D_{i}^{d}(\bmod N)$
$U_{i}\left(\right.$ or $\left.P_{i}\right)$

Select: $I D_{i}$ $\underset{\text { (Secure Channel) }}{\stackrel{S_{i}}{\longrightarrow} \quad \text { Secret token: } S_{i}}$

Fig. 1. Key Generation Phase of WH scheme

(2) After receiving the request, $P_{j}$ chooses a random number $k$ and computes $z$, where $z=g^{k} S_{j}(\bmod N)$. Then, $P_{j}$ sends $z$ to $U_{i}$.

(3) $U_{i}$ randomly chooses a number $t$ and computes $a, x$, and $y$, where $a=$ $z^{e} / I D_{j}(\bmod N), x=S_{i} h\left(a^{t} \| T\right)(\bmod N), y=g^{e t}(\bmod N)$, and $T$ is the timestamp. Then, $U_{i}$ sends $(x, y, T)$ to $P_{j}$.

(4) Finally, $P_{j}$ checks $T$ and verifies the equality $I D_{i} \stackrel{?}{=}\left(x / h\left(y^{k} \| T\right)\right)^{e}(\bmod N)$. If it holds for some $I D_{i}$ existing in the identity list, $U_{i}$ is accepted as an authorized user and the service request will be granted.

The user and the service provider share common session key as $k_{i j}=a^{t x}=$ $y^{k x}=g^{e k t x}(\bmod N)$, which can be used in subsequent communications for confidentiality.

$$
\begin{array}{ccc}
U_{i} & & P_{j} \\
& \begin{array}{c}
\text { (Service } \\
\text { request) }
\end{array} & k \in Z_{N}^{*} \\
a \leftarrow z^{e} / I D_{j}(\bmod N) & \stackrel{z}{ } & z \leftarrow g^{k} S_{j}(\bmod N) \\
t \in Z_{N}^{*} & & \\
x \leftarrow S_{i} h\left(a^{t} \| T\right)(\bmod N) & & \text { Check: } T \\
y \leftarrow g^{e t}(\bmod N) & \stackrel{(x, y, T)}{ }
\end{array}
$$$$
\stackrel{(x, y, T)}{\longrightarrow}
$$$$
\text { Check: } T
$$

Verify: $I D_{i} \stackrel{?}{=}\left(x / h\left(y^{k} \| T\right)\right)^{e}(\bmod N)$

Fig. 2. Anonymous User Identification Phase of WH scheme 


\subsection{YWBWD Scheme}

To solve the security problem in the WH scheme, Yang et al. proposed an improved version of the WH scheme. The YWBWD scheme is also composed of two phases; key generation and anonymous user identification.

Key Generation Phase: The key generation phase in the YWBWD scheme is similar to that of the WH scheme. The key generation phase of YWBWD scheme, which is illustrated in Figure 3, is as follows: The smart card producing center $(S C P C)$ first chooses two large primes $p$ and $q$, computes $N=p q$, picks an element $g \in Z_{N}^{*}$ (which is the generator of both $Z_{p}$ and $Z_{q}$ ) and a hash function $h(\cdot)$, and selects $e$ and $d$ such that $e d=1(\bmod \phi(N))$, where $\phi(N)(=$ $(p-1)(q-1))$ is the Euler totient function. Note that $e$ must be sufficiently large, e.g., 160 bits. Additionally, SCPC picks a symmetric-key cryptosystem such as DES Schneier, 1996, whose encryption function and decryption function under the private key $K$ are $E_{K}(\cdot)$ and $D_{K}(\cdot)$, respectively. $N, e, g$, and $h(\cdot)$ are published and $d, p$, and $q$ are kept secret by $S C P C$. Then, $S C P C$ sends each user $U_{i}$ (or service provider $P_{i}$ ) a secret token $S_{i}$ with a secure channel, where $S_{i}=I D_{i}^{d}(\bmod N)$ and $I D_{i}$ is the identity of $U_{i}\left(\right.$ or $\left.P_{i}\right)$.

Anonymous User Identification Phase: The anonymous user identification phase of the YWBWD scheme, which is illustrated in Figure 4, is as follows:

(1) $U_{i}$ first submits a service request to $P_{j}$ to request a service from the service provider $P_{j}$.

(2) After receiving the request, $P_{j}$ chooses a random number $k$ and computes $z$, where $z=g^{k} S_{j}^{-1}(\bmod N)$. Then, $P_{j}$ sends $z$ to $U_{i}$.

(3) $U_{i}$ randomly chooses a number $t$ and computes $a, K_{i j}, x, s$, and $y$, where $a=$ $z^{e} I D_{j}(\bmod N), K_{i j}=a^{t}(\bmod N), x=g^{e t}(\bmod N), s=g^{t} S_{i}^{h(x, T)}(\bmod N)$,

\section{$S C P C$}

Choose: $p, q$

Compute: $N=p q$

Pick: $g \in Z_{N}^{*}, h(\dot{)}$

Compute: $\phi(N)=(p-1)(q-1)$

Select: $e, d(e d=1(\bmod \phi(N)))$

Pick: $E_{K}(\cdot), D_{K}(\cdot)$

Publish: $N, e, g, h(\cdot)$

Keep secret: $d, p, q$

Compute: $S_{i}=I D_{i}^{d}(\bmod N)$ $\underset{(\text { Secure Channel) }}{\stackrel{S_{i}}{\longrightarrow}}$
$U_{i}\left(\right.$ or $\left.P_{i}\right)$

Select: $I D_{i}$

Fig. 3. Key Generation Phase of YWBWD scheme 


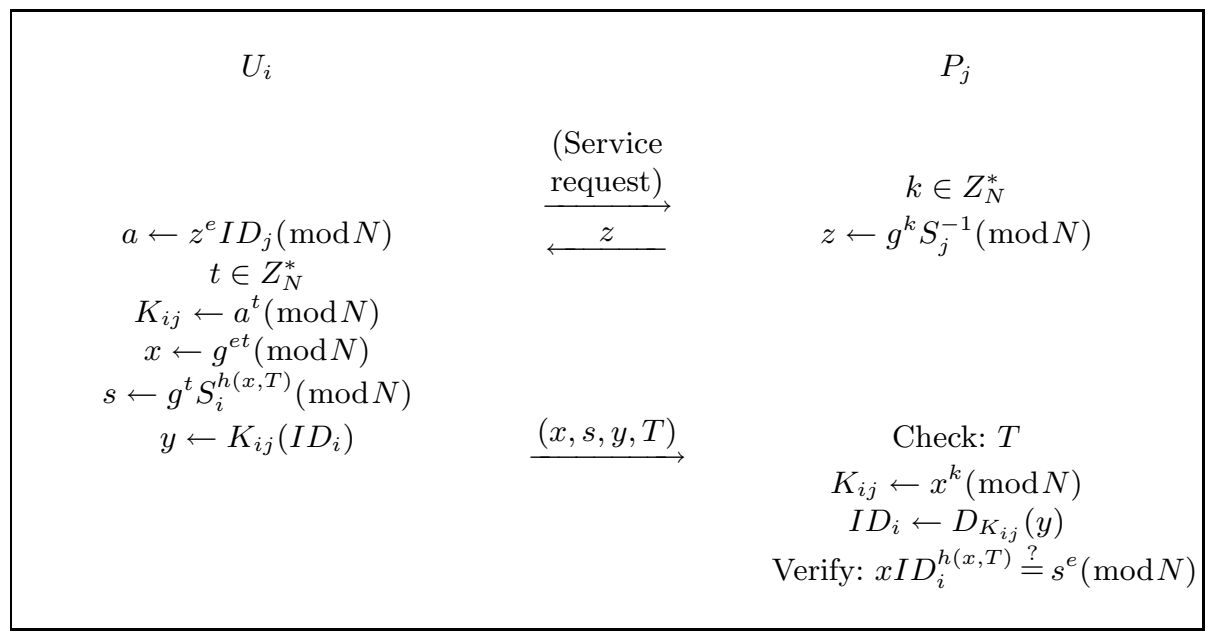

Fig. 4. Anonymous User Identification Phase of YWBWD scheme

$y=K_{i j}\left(I D_{i}\right)$, and $T$ is the timestamp. Then, $U_{i}$ sends $(x, s, y, T)$ to $P_{j}$. Note that $K_{i j}$ is the common session key.

(4) Finally, $P_{j}$ first checks $T$. If it is old, $P_{j}$ aborts the protocol. Otherwise, $P_{j}$ obtains the common session key $K_{i j}=x^{k}(\bmod N)$. With $K_{i j}, P_{j}$ proceeds to decrypt $y$ as $I D_{i}=D_{K_{i j}}(y) . P_{j}$ then checks whether $I D_{i}$ is on his maintained list. If $I D_{i}$ is a legitimate user, $P_{j}$ verifies the equality $x I D_{i}^{h(x, T)} \stackrel{?}{=} s^{e}(\bmod N)$. If the verification passes, then the service request is granted. Otherwise, the request is rejected.

The user and the service provider share common session key as $k_{i j}=a^{t}=$ $x^{k}=g^{e k t}(\bmod N)$, which can be used in the subsequent communications for confidentiality.

\section{Cryptanalysis of Two Schemes}

This section show the security flaws of the WH scheme and YWBWD scheme. In the schemes, an attacker can freely impersonate the users or the service provider. This happens because an attacker can obtain the secret token $S_{i}\left(S_{j}\right)$ of the user (or the service provider) after successful execution of the key generation phase.

\subsection{Attack to $U_{i}$}

Suppose user $U_{f}$ is an attacker who knows the legal user $U_{i}$ 's $I D_{i}$. Usually, because the legal user's $I D_{i}$ does not require safety, an attacker can easily get the target user's $I D_{i}$ by various attack methods, such as stolen-verifier attacks [7] and server data eavesdropping [8]. For example, service provider $P_{j}$ is always the target of attacker, because numerous users' secrets are stored in their databases. 
The user $I D$ table list stored in the service provider $P_{j}$ can be eavesdropped and then used to impersonate the original user. By using the legal user $U_{i}$ 's $I D_{i}$, in the key generation phase, $U_{f}$ can register with $S C P C$ as follows:

(1) $U_{f}$ obtains his/her identity $I D_{f}$ by $I D_{f}=I D_{i}^{-1}$ and submits $I D_{f}$ as registration request to $S C P C$.

(2) $S C P C$ will compute the secret token $S_{f}$ of $U_{f}$ by $S_{f}=I D_{f}^{d}=I D_{i}^{-d}=$ $S_{i}^{-1}(\bmod N)$ and send $S_{f}$ to $U_{f}$ with a secure channel.

As a result, $U_{f}$ can obtain the secret token $S_{i}$ of the legal user $U_{i}$ by computing $S_{f}^{-1}=S_{i}(\bmod N)$. Then, by using the $S_{i}$, so obtained, $U_{f}$ can freely impersonate $U_{i}$ to request a service from $P_{j}$ and thus gain access privilege.

\subsection{Attack to $P_{j}$}

Suppose user $U_{f}$ is an attacker who knows the the service provider $P_{j}$ 's $I D_{j}$. In the key generation phase, $U_{f}$ can register with $S C P C$ as follows:

(1) $U_{f}$ obtains his/her identity $I D_{f}$ by $I D_{f}=I D_{j}^{-1}$ and submits $I D_{f}$ as registration request to $S C P C$.

(2) $S C P C$ will compute the secret token $S_{f}$ of $U_{f}$ by $S_{f}=I D_{f}^{d}=I D_{j}^{-d}=$ $S_{j}^{-1}(\bmod N)$ and send $S_{f}$ to $U_{f}$ with a secure channel.

As a result, $U_{f}$ can obtain the secret token $S_{j}$ of the service provider $P_{j}$ by computing $S_{f}^{-1}=S_{j}(\bmod N)$. Then, by using obtained $S_{j}, U_{f}$ can impersonate $P_{j}$ and exchange a common session key with legal user $U_{i}$.

\subsection{Another Attack}

As another attack on two schemes, if a malicious $U_{i}$ or $P_{j}$, who knows his/her $S_{i}$ or $S_{j}$, computes his/her new identity $I D_{f}$ by $I D_{f}=I D_{i} I D_{j}$ and resubmits $I D_{f}$ as a registration request to $S C P C$. Then, $S C P C$ will compute the secret token $S_{f}$ of $U_{f}$ by $S_{f}=I D_{f}^{d}=\left(I D_{i} I D_{j}\right)^{d}=S_{j} S_{j}(\bmod N)$ and send $S_{f}$ to $U_{f}$ with a secure channel. Consequently, a malicious $U_{i}$ can obtain the secret token $S_{i}$ of the legal user $U_{i}$ or $S_{j}$ of the service provider $P_{j}$ by computing $S_{i}=S_{f} S_{j}^{-1}(\bmod N)$ or $S_{j}=S_{f} S_{i}^{-1}(\bmod N)$, respectively.

\section{Improved Scheme}

This section presents a modification of the two schemes to correct the security flaws described in Section 3.

The proposed scheme employs the concept of hiding identity to prevent from above attacks. We only modify the key generation phase which issues a "hashed" identity for every legal user. That is, in the key generation phase, the smart card 
$S C P C$

Choose: $p, q$

Compute: $N=p q$

Pick: $g \in Z_{N}^{*}, h(\dot{)}$

Compute: $\phi(N)=(p-1)(q-1)$

Select: $e, d(e d=1(\bmod \phi(N)))$

Pick: $E_{K}(\cdot), D_{K}(\cdot)$

Publish: $N, e, g, h(\cdot)$

Keep secret: $d, p, q$

Compute: $S_{i}=H I D_{i}^{d}(\bmod N)$ $\underset{\text { (Secure Channel) }}{\stackrel{S_{i}}{\longrightarrow}}$
$U_{i}\left(\right.$ or $\left.P_{i}\right)$

Select: $I D_{i}$

Compute: $H I D_{i}=h\left(I D_{i}\right)$

Fig. 5. Proposed Key Generation Phase

producing center $\left(S C P C\right.$ ) sends each user $U_{i}$ (or service provider $P_{i}$ ) a secret token $S_{i}=H I D_{i}^{d}(\bmod N)$ with a secure channel, where $H I D_{i}=h\left(I D_{i}\right)$. The steps of the anonymous user identification phase are retained except that $I D_{i}$ is replaced by "hashed" identity $H I D_{i}$, respectively. The proposed key generation phase is illustrated in Figure 5.

\section{Security Analysis}

This section discusses the enhanced security features. The rest are the same as the original YWBWD scheme as described in the literature [6]. Readers are referred to [6] for completer references.

Definition 1. One-way hash function assumption [349]: Let $h(\cdot)$ be an oneway cryptographic hash function, (1) given $y$, it is computationally intractable to find $x$ such that $y=h(x)$; (2) it is computationally intractable to find $x_{1} \neq x_{2}$ such that $h\left(x_{1}\right)=h\left(x_{2}\right)$.

Theorem 1. In the proposed key generation phase, an illegal user cannot get the legal user or service provider's secret token $S_{i}$.

Proof. The attacks on WH scheme and YWBWD scheme works because a malicious user can successfully register a new $I D_{f}$ via $I D_{i}$ or $I D_{j}$ in the key generation phase. In our improved key generation phase, since the format of $H I D_{f}^{d}=h\left(I D_{i}^{-1}\right)^{d}(\bmod N)\left(\right.$ or $\left.h\left(I D_{i} I D_{j}\right)^{d}(\bmod N)\right)$ is not equal to $I D_{f}^{d}=I D_{i}^{-d}(\bmod N)\left(\right.$ or $\left.S_{i} S_{j}(\bmod N)\right)$, a malicious user cannot get the legal user or service provider's secret token $S_{i}$. Therefore, the proposed scheme can correct the security flaws described in Section 3 . 


\section{Conclusions}

The current paper demonstrated the security flaws of the WH user identification scheme and YWBWD user identification scheme. Using our attacks, we have shown that a malicious user (including a service provider) can easily get a specific legal user's secret token and impersonate this specific user to request a service from the service provider and gain access privilege. Additionally, we have shown that a malicious user (including the legal user) can easily get the service provider's secret token and impersonate this service provider to exchange a common session key with a legal user. For the above attacks, we presented an improvement to repair the security flaws of the two schemes.

\section{Acknowledgements}

We would like to thank the anonymous reviewers for their helpful comments in improving our manuscript. This research was supported by the MIC (Ministry of Information and Communication), Korea, under the ITRC (Information Technology Research Center) support program supervised by the IITA (Institute of Information Technology Assessment).

\section{References}

1. Lee, W.B., Chang, C.C.: User Identification and Key Distribution Maintaining Anonymity for Distributed Computer Network. Computer Systems Science and Engineering. Vol. 15. No. 4. (2000) 113-116

2. Rivest, R., Shamir, A., Adleman, L.: A Method for Obtaining Digital Signature and Public-key Cryptosystem. Commun ACM. Vol. 21. No. 2. (1978) 120-126

3. Schneier, B.: Applied Cryptography. 2nd ed. John Wiley \& Sons. Inc. (1996)

4. Diffie, W., Hellman, M.: New Directions in Cryptography. IEEE Trans Inf Theory. Vol. 22. No. 6. (1976) 644-654

5. Wu, T.S., Hsu, C.L.: Efficient User Identification Scheme with Key Distribution Preserving Anonymity for Distributed Computer Networks. Computer \& Security. Vol. 23. No. 2. (2004) 120-125

6. Yang, Y.J., Wang, S.H., Bao, F., Wang, J., Deng, R.H.: New Efficient User Identification and Key Distribution Scheme Providing Enhanced Security. Computer \& Security. Vol. 23. No. 8. (2004) 697-704

7. Lin, C.L., Hwang, T.: A Password Authentication Scheme with Secure Password Updating. Computers \& Security. Vol. 22. No. 1. (2003) 68-72

8. Yang. C.C., Chang. T.Y., Li, J.W.: Security Enhancement for Protecting Password Transmission. IEICE Transactions on Communications. Vol. E86-B. No. 7. (July 2003) 2178-2181

9. Menezes, A.J., Oorschot, P.C., Vanstone, S.A.: Handbook of Applied Cryptograph. CRC Press. New York. (1997) 